\section{DNMT1 regulates IL-6- and TGF- $\beta 1$-induced epithelial mesenchymal transition in prostate epithelial cells}

\author{
Hui Xu, ${ }^{1}$ Yanbo Chen, ${ }^{2}$ Qi Chen, ${ }^{2}$ \\ Huan Xu, ${ }^{2}$ Yanxia Wang, ${ }^{1}$ Jiao Yu, ${ }^{1}$ \\ Juan Zhou, ${ }^{2}$ Zhong Wang, ${ }^{2}$ Bing $\mathrm{Xu}^{1}$ \\ ${ }^{1}$ Department of Emergency, Shanghai \\ Ninth People's Hospital Affiliated to \\ Shanghai Jiaotong University School of \\ Medicine, Shanghai \\ ${ }^{2}$ Department of Urology, Shanghai Ninth \\ People's Hospital Affiliated to Shanghai \\ Jiaotong University School of Medicine, \\ Shanghai, China
}

\section{Introduction}

Benign prostatic hyperplasia (BPH) resulting in lower urinary tract symptoms is a common disease developing in $50 \%$ of men older than 50 years. Multiple factors have been considered to play roles in the development of $\mathrm{BPH}$, including chronic inflammation, ${ }^{1,2}$ hormones, dietary factors, and local endocrine and autonomous nerve system deregulation. ${ }^{3}$ However, few studies have focused on the role of epigenetic factors. Typically, DNA methylation has been recognized to regulate gene expression by suppressing transcription. DNA methylation was catalyzed by DNA methyltransferases (Dnmts), which are classified as maintenance (Dnmt1) and de novo methyltransferases (Dnmt3a, Dnmt3b). ${ }^{4,5}$ Recently, interplay between DNA methylation and prostate-associated diseases has been found. The most studied 5-alpha reductase, SRD5A2, has CpG regions, and its expression, which is varied in adulthood, is regulated by Dnmt $1 .{ }^{6}$ Increased methylation of the SRD5A2 promoter is associated with increasing age in humans and with decreased expression. ${ }^{7}$ Thus, DNA methylation could be a personalized medical target for the management of BPH patients resistant to 5-alpha reductase inhibitor therapy. ${ }^{8}$

In addition, epithelial-mesenchymal transition (EMT), a process by which epithelial cells lose their cell polarity and cell-cell adhesion, such as E-cadherin, and they convert to motile mesenchymal cells, ${ }^{9}$ has often been identified to act during the initiation and progression of $\mathrm{BPH} .{ }^{10-12}$ In $\mathrm{BPH}$ samples, the accumulation of mesenchymal-like cells derived from the prostatic epithelium was observed. ${ }^{11}$
Inflammation is regarded as one of the potential inducers of EMT. It was shown that the E-cadherin protein was down-regulated in BPH1 cells after transforming growth factor- $\beta 1$ (TGF- $\beta 1$ ) treatment, whereas $\mathrm{N}$-cadherin expression was increased. ${ }^{13}$ Interleukin 6 (IL-6), an essential cytokine participating in inflammation and $\mathrm{BPH}$, has also been reported to induce EMT in a number of tumor cell types. ${ }^{14}$ However, the mechanisms that modulate pro-inflammatory factors (IL-6 and TGF$\beta 1$ ) inducing some EMT characteristic, have not yet been studied in the prostate. Thus, in this report, we speculated that IL-6 and TGF- $\beta 1$ could change E-cadherin expression by directly regulating DNMT1 and thus the DNA methylation status of the Cdh1 promoter region. Indeed, our results showed that IL- 6 and TGF- $\beta 1$ decreased Ecadherin expression and increased the expression and activity of DNMT1, which in turn induced DNA hypermethylation in the promoter region of Cdh1. Moreover, we found that the combination of the DNMT1 inhibitor 5-aza-2'-deoxycytidine (5-Aza) and TGF- $\beta 1$ antibody significantly rescued E-cadherin expression, providing a promising strategy for the treatment of BPH.

\section{Materials and Methods}

\section{Ethical statements}

All involved subjects gave their informed consent in the study. The study was conducted in accordance with the Declaration of Helsinki, and the protocol was approved by the Ethics Committee of Shanghai Ninth Hospital (2012KY-035).

\section{Cell cultures and treatment}

BPH-1 cells, derived from $\mathrm{BPH}$ patients, were obtained from the Shanghai Jiao Tong University. The cells were cultured in RPMI 1640 medium supplemented with $20 \%$ fetal bovine serum (Hyclone, Thermo Fisher Scientific, Waltham, MA, USA), $1 \%$ penicillin, and streptomycin (Corning, Manassas, VA, USA). Human normal prostate epithelial cells (PrECs; at passage 2) were purchased from Lonza (Allendale, NJ, USA) and were cultured according to the supplier. ${ }^{15}$ The cells were grown under standard culture conditions of $5 \% \mathrm{CO}_{2}$ at $37^{\circ} \mathrm{C}$.

For the transfection of siRNA oligos, the culture medium was replaced with FBSfree transfection medium. The specific siRNA for Cdh1 was transfected at a concentration of $50 \mathrm{nM}$. The cells were trans-
Correspondence: Zhong Wang, Department of Urology, Shanghai Ninth People's Hospital Affiliated to Shanghai Jiaotong University School of Medicine, Shanghai 200011, China. Fax: +86.21.63136856.

E-mail: zhongwang2010@sina.com

Bing Xu, Department of Emergency, Shanghai Ninth People's Hospital Affiliated to Shanghai Jiaotong University School of Medicine, Shanghai 200011, China.

Fax: +86.21.63136856.

E-mail: saint_xue@msn.com

Hui Xu, Yanbo Chen and Qi Chen contributed equally to this work.

Keywords: Benign prostatic hyperplasia; EMT; DNMT1; methylation.

Conflict of interest: the authors declare no conflict of interest.

Acknowledgments: this study was supported by the Program of Shanghai City Committee of Science and Technology (15DZ1941502); key disciplines group construction project of Pudong Health Bureau of Shanghai (PWZxq2014-11).

Received for publication: 28 January 2017. Accepted for publication: 20 March 2017.

This work is licensed under a Creative Commons Attribution-NonCommercial 4.0 International License (CC BY-NC 4.0).

CCopyright H. Xu et al., 2017

Licensee PAGEPress, Italy

European Journal of Histochemistry 2017; 61:2775 doi:10.4081/ejh.2017.2775

fected using Lipofectamine ${ }^{\mathrm{TM}} 2000$ transfection reagent (Invitrogen, Carlsbad, CA, USA), according to the manufacturer's instructions. The siRNA sequence was as follows: siRNA-Cdh1: GGCCTGAAGTGACTCGTAATT; siRNA-Dnmt1: CGGUGCUCAUGCUUACAACTT.

\section{EdU incorporation assay}

Cell proliferation was detected using a Click-iT EdU (5-ethynyl- 2'-deoxyuridine) Alexa Fluor 488 Imaging Kit (Invitrogen) according to the manufacturer's instructions. Fluorescence was analyzed using a Zeiss 510 laser-scanning microscope (Zeiss, Thornwood, NY, USA).

\section{Annexin V/propidium iodide staining}

Cell apoptosis was assessed by flow cytometry using the Alexa Fluor 488 Annexin V/Dead Cell Apoptosis Kit (Invitrogen). The samples were analyzed directly by flow cytometry using the Cell Quest program (BD Biosciences, San Jose, CA, USA). 


\section{Quantitative real-time PCR}

The total RNA of cells was isolated using TRIzol reagent (Invitrogen), according to the manufacturer's instructions. Purified RNA was subjected to reverse transcription with Oligo (dT) and M-MLV Reverse Transcriptase (Thermo Fisher Scientific). Synthesized complementary DNA (cDNA)was analyzed by real-time polymerase chain reaction (PCR), performed with an ABI 7900HT system using SYBR $^{\circledR}$ Premix (Takara, Dalian, China), according to the manufacturer's instructions. The conditions of the real-time PCR were as follows: denaturation at $9{ }^{\circ} \mathrm{C}$ for 10 s, 45 cycles at $95^{\circ} \mathrm{C}$ for $10 \mathrm{~s}$, and $65^{\circ} \mathrm{C}$ for 30 s. Glyceraldehyde-3-phosphate dehydrogenase (GAPDH) was used as the reference gene. No nonspecific amplification was observed. The data were analyzed using the comparison $\mathrm{Ct}\left(2^{-\Delta \Delta \mathrm{Ct}}\right)$ method and were expressed as fold changes relative to the respective controls.

The primer sequences were as follows:

Cdh1: forward, 5'-CGAGAGCTACACG TTCACGG-3'; reverse, 5'-GGGTGTCGA GGGAAAAATAGG-3'; Vimentin: forward, 5'-GACGCCATCAACACCGAGTT-3'; reverse, 5'-CTTTGTCGTTGGTTAGCTGGT-3'; MMP9: forward, 5'-GGGACGCAGACATCGTCATC-3'; reverse, 5'-TCGTCATCGTCGAAATGGGC-3'; IL-6: forward, 5'-ACTCACCTCTTCAGAACGAATTG-3'; reverse, 5'-CCATCTTTGGAAGGTTCAGGTTG-3'; TGF- $\beta 1$ : forward, 5'-CAATTCCTGGCGATACCTCAG-3'; reverse, 5'-GCACAACTCCGGTGACATCAA-3'; Dnmt1: forward, 5'-AGAACGGTGCTCATGCTTACA-3'; reverse, 5'-CTCTACGGGCTTCACTTC TTG-3'. And GAPDH: forward, 5'CCTCTGACTTCAACAGCGAC-3'; reverse, 5'-TCCTCTTGTG CTCTTGCTGG-3'.

Each sample was analyzed in triplicate.

\section{ELISA}

IL-6 and TGF- $\beta 1$ levels were measured using Quantikine human ELISA kits from R\&D Systems (Minneapolis, MN, USA), according to the manufacturer's instructions. Levels of IL- 6 and TGF- $\beta 1$ proteins in the culture supernatants were expressed as fold increases, normalized by the total proteins. Each test was performed in triplicate.

\section{Western blot}

The cells were lysed using radioimmunoprecipitation assay (RIPA) lysis buffer supplemented with protease inhibitors (10 $\mathrm{mg} / \mathrm{mL}$ leupeptin, $10 \mathrm{mg} / \mathrm{mLl}$ pepstatin A, and $10 \mathrm{mg} / \mathrm{mL}$ aprotinin). The protein concentrations were determined using a micro bicinchoninic acid (BCA) assay (Thermo Fisher Scientific). Twenty micrograms of total protein extract were separated by $10 \%$ sodium dodecyl sulfate-polyacrylamide gel electrophoresis (SDS-PAGE) under reducing conditions and were transferred to polyvinylidene difluoride (PVDF) membranes. The membranes were blocked with $5 \%$ BSA and were then incubated with specific antibodies overnight at $4^{\circ} \mathrm{C}$. A horseradish peroxidase-labeled secondary antibody was used and visualized using an enhanced chemiluminescence detection system (Millipore, Billerica, MA, USA), as recommended by the manufacturer. The primary antibodies used included the following: anti-E-cadherin: 1:1000 (Cell Signaling Technology, Inc.); anti-Vimentin: 1:500 (Abcam, Cambridge, UK); anti-MMP9: 1:1000 (Cell Signaling Technology, Inc.); anti-DNMT1: 1:1000 (Cell Signaling Technology, Inc.); and anti- $\beta$-actin: 1:2500 (Thermo Fisher Scientific).

\section{Cell migration assessment}

Cell migration was assessed using the Oris Cell Migration Assay (AMSbio, Oxford, UK. A total of $5^{\prime} 10^{4}$ cells were seeded into a 96-well plate containing well bottoms coated with collagen. The cell seeding stopper restricted the seeded cells to the outer regions of the wells. Removal of the stopper resulted in a round, unseeded region, into which the seeded cells could migrate. The cells were then incubated withIL- 6 and TGF- $\beta 1$ and were stained with the acetomethoxy derivative of calcein (Calcein AM; Abcam) for $30 \mathrm{~min}$. Cell migration was assessed using an FLx 800 microplate fluorescence reader (Biotek Instruments, Winooski, VT, USA).

\section{Immunofluorescence}

The cells were fixed with paraformaldehyde and blocked with 5\% goat serum in PBST (PBS with $0.1 \%$ TritonX-100) for 1 h. Incubation with the primary anti-E-cadherin antibody (1:250, Abcam) was performed overnight at $4{ }^{\circ} \mathrm{C}$, followed by an Alexa Fluor 488-conjugated secondary antibody. The nuclei were counterstained by 4',6-diamidino-2-phenylindole (DAPI; Sigma-Aldrich, St. Louis, MO, USA) for 10 min before imaging. Immunofluorescence signals were captured using confocal microscopy (Leica, Solms, Germany).

\section{DNA methylation analysis - bisulfite sequencing PCR, BSP}

The cells were lysed in lysis buffer (100 $\mathrm{mM}$ EDTA, $0.5 \%$ SDS, $10 \mathrm{mM}$ Tris- $\mathrm{HCl}$,
$\mathrm{pH} 8$ ) for $10 \mathrm{~min}$ and were digested with 0.1 $\mathrm{mg} / \mathrm{mL}$ proteinase $\mathrm{K}$ overnight. Bisulfite conversion was performed as previously described (16). Briefly, $1 \mathrm{mg}$ of genomic DNA was treated with $123 \mathrm{mM}$ hydroquinone and $2.4 \mathrm{M}$ sodium bisulfite (both from Sigma-Aldrich). The reactions were desalted using a QIAEX II gel extraction kit (Qiagen). Converted DNA samples were amplified with primers (from -185 bp to -57 bp upstream of the transcription start sites) in the $\mathrm{CpG}$ island region of $\mathrm{Cdh} 1$. The primer sequences were as follow: sense, 5'TAGTAATTTTAGGTTAGAGGGTTAT3'; and antisense, 5'-CTAATTAACTAAAAATTCACCTACC-3').

PCR products were cloned intopMD19$\mathrm{T}$ vector (TaKaRa, Dalian, China); thereafter, ten clones isolated from a PCR amplicon were sequenced.

\section{Nuclear protein extraction and DNMT activity}

Nuclear proteins were extracted using the EpiQuik Nuclear Extraction kit (EpigenTek). Total Dnmt1 activity in nuclear extracts was determined using the EpiQuik Dnmt Activity Assay Ultra kit (EpigenTek). DNMT1 activity was calculated as $\mathrm{OD} / \mathrm{h} / \mathrm{mg}$ of protein.

\section{Statistical analysis}

The data are presented as the mean $\pm \mathrm{sd}$ ( $\mathrm{n}$ is the number of experimental replicates). To compare groups of data, Student's twotailed $t$-test was used. A value of $\mathrm{P}<0.05$ was considered to be statistically significant. Statistical analysis was performed using GraphPad Prism (ver. 5.0) software.

\section{Results}

\section{Expression of EMT features is increased in BPH1 cells}

We compared the expression profiles of normal human prostate epithelial cells (PrECs) with BPH1 cells. Genome-wide gene expression patterns were examined using microarray hybridizations (the microarrays were downloaded from the GEO database, GEO accession numbers: GSM88411, GSM88412, GSM88413 for PrECs; GSM2139697, GSM2139698, GSM2139699 for BPH1). Using a heat map for genes that were differentially expressed by at least 2-fold between the PrECs and BPH1 cells, we found that the BPH1 cells exhibited a different gene expression profile, in which the features of EMT, Vimentin, MMP9, Snai2, N-cadherin, TGF$\beta 1$ and AR (androgen receptor), were upreg- 
ulated (Figure 1a). Then, the mRNA and protein expression of EMT features was investigated. As shown in Fig. 1b, compared with PrECs, the mRNA expression of Vimentin and MMP9 was up-regulated in BPH1 cells with Cdh1 down-regulated. These RT-PCR results were further confirmed using western blot analysis (Figure 1c), and the band intensities of E-cadherin,
Vimentin and MMP9 were quantified (Figure 1d). By EdU incorporation assay of cell proliferation and annexin V/PI detection of apoptosis, no significant difference was found between PrECs and BPH1 cells (Figure $1 \mathrm{e}-\mathrm{g}$ ). These results clearly indicated that BPH1 cells showed some EMT features, consistent with previous reports. ${ }^{11}$

\section{IL-6 and TGF- $\beta 1$ induce EMT fea- tures by decreasing E-cadherin expression}

Since pro-inflammation cytokines were found to induce EMT features, we examined the expression of the acknowledged EMT inducers IL-6 and TGF- $\beta 1$. First, the IL-6 and TGF- $\beta 1$ mRNA levels were a

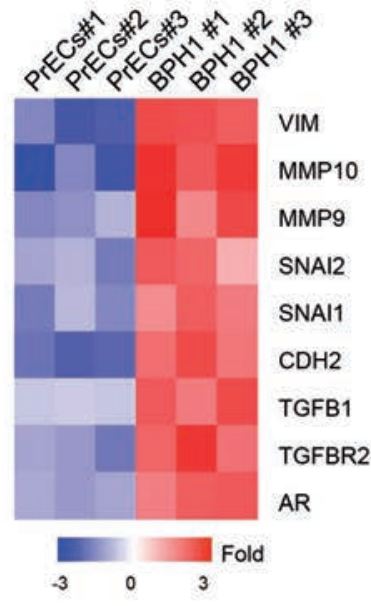

d

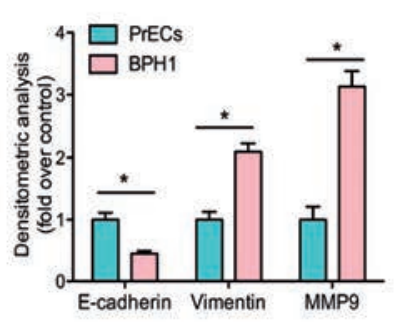

b

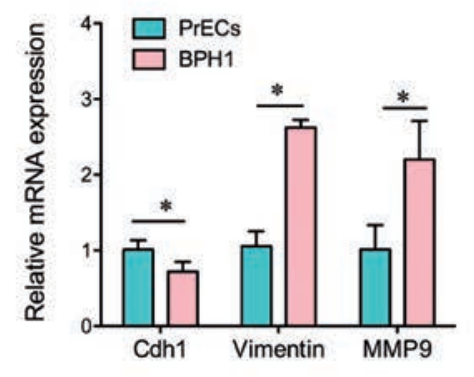

c

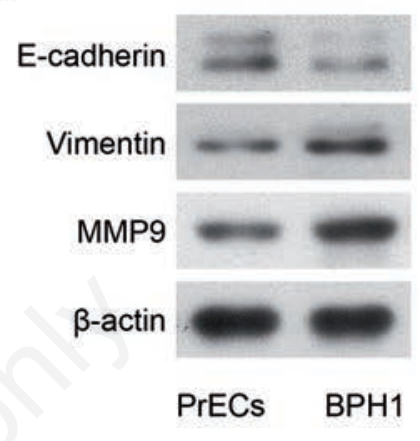

e

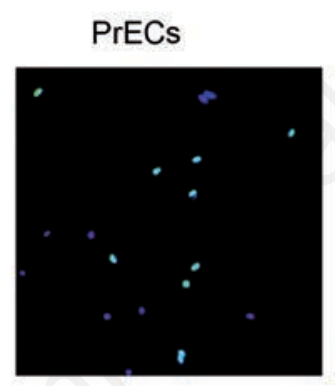

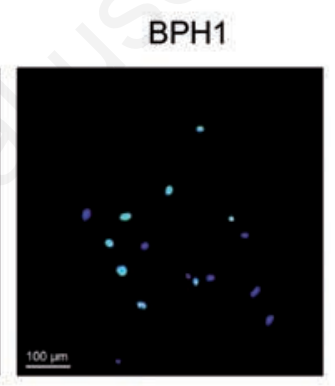

f

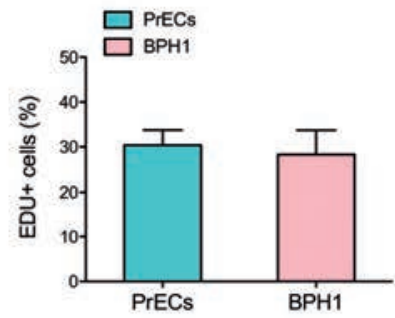

g
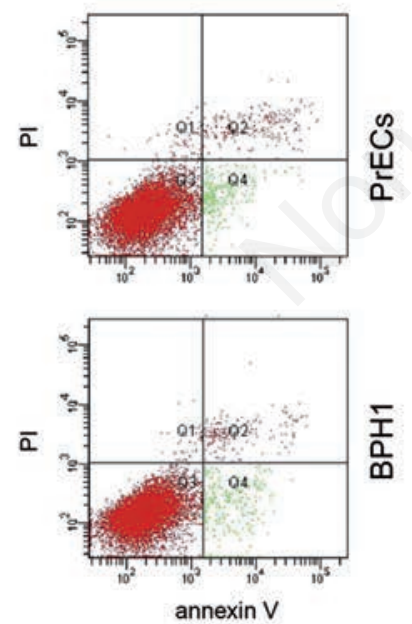

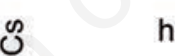

h

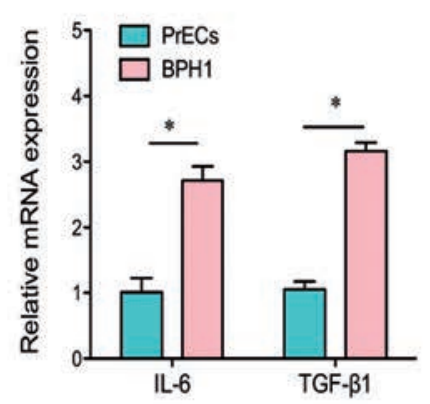

i

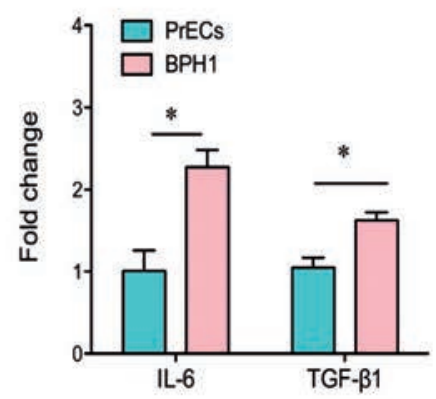

Figure 1. The expression levels of EMT features are increased in BPH1 cells. a) The heat map is shown by the degree of differential expression of EMT feature genes between PrECs and BPH1 cells. b) Quantitative real-time PCR analysis (qRT-PCR) of Cdh1, Vimentin, and MMP9 mRNA levels in PrECs and BPH1 cells; GAPDH was used as internal control. c) Western blot showed the expression of Ecadherin, Vimentin, and MMP9 protein levels in PrECs and BPH1 cells. d) The band intensity corresponding to E-cadherin, Vimentin, and MMP9 were quantified and normalized to -actin using ImageJ software. e) 5-Ethynyl-2 -deoxyuridine (EdU, green) was used to label proliferating cells, while the nucleus was stained with 4 ,6-diamidino-2-phenylindole (blue); scale bar: $50 \mathrm{~m}$. f) Quantification of the percentage of EdU-positive cells. g) Apoptosis was analyzed and examined by annexin V/PI fluorescence by fluorescence-activated cell sorting analysis. h) qRT-PCR analysis and (i) ELISA of the expression ofIL-6 and TGF- $\beta 1$ in PrECs and BPH1 cells. All of the results are representative of at least three independent experiments. The data are expressed as the mean $\pm S D,{ }^{*} P<0.05$. All of the $P$ values were based on Student's $t$-test. 
increased in BPH1 cells, compared to PrECs (Figure 1h), as confirmed by the ELISA results (Figure 1i). Next, PrECs were exposed to IL-6 (50 ng/mL) and TGF$\beta 1(5 \mathrm{ng} / \mathrm{mL})$ and were harvested for RTPCR, ELISA and western blot analysis. It was shown by RT-PCR that, after treatment with IL- 6 and TGF- $\beta 1$ for $72 \mathrm{~h}$, the mRNA expression of Cdh1 was decreased, whereas
Vimentin and MMP9 were obviously upregulated (Figure $2 \mathrm{a}, \mathrm{b}$ ). These results were confirmed by Western blot analysis (Figure $2 \mathrm{c}, \mathrm{e})$, and the band intensities of E-cadherin, Vimentin and MMP9 were quantified (Figure 2 d,f). Then, by immunofluorescence, E-cadherin levels could be clearly found to be decreased when exposed to IL6 and TGF- $\beta 1$ for $72 \mathrm{~h}$ (Figure $2 \mathrm{~g}$ ). To study the cell migration of PrECs, the cells were allowed to migrate on collagen coating. The results of cell migrating on collagen coating were significantly promoted after exposure to IL- 6 and TGF- $\beta 1$ for $72 \mathrm{~h}$ (Figure $2 \mathrm{~h}, \mathrm{i}$ ). These results indicated that IL- 6 and TGF$\beta 1$ induced EMT features, especially the down-regulation of E-cadherin in PrECs. a

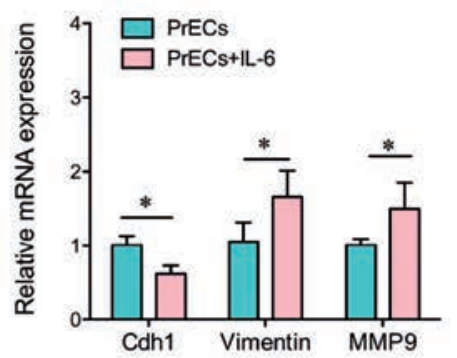

c

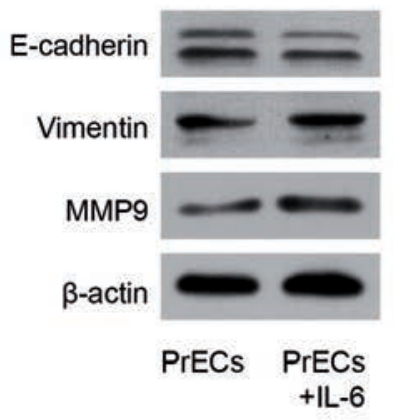

g

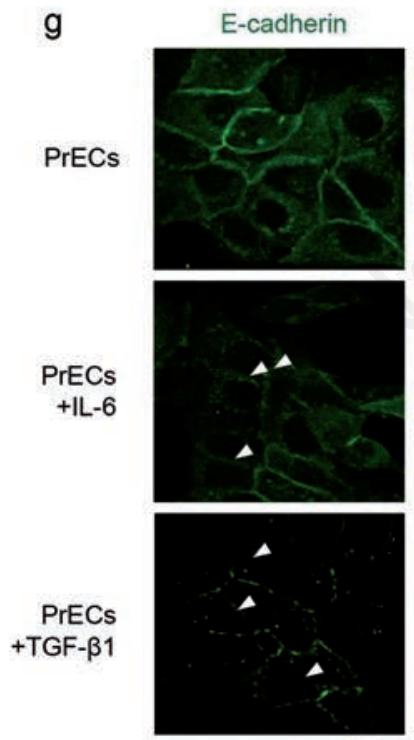

PrECs

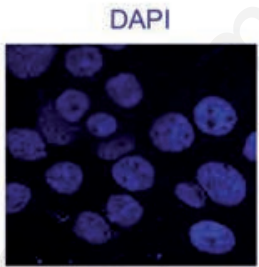

b

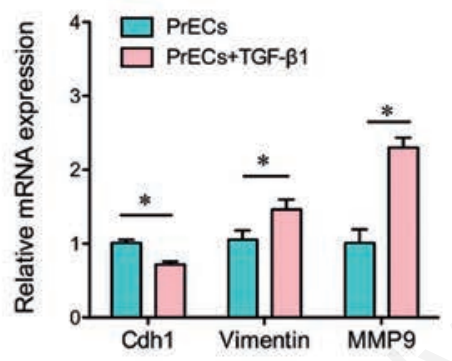

e

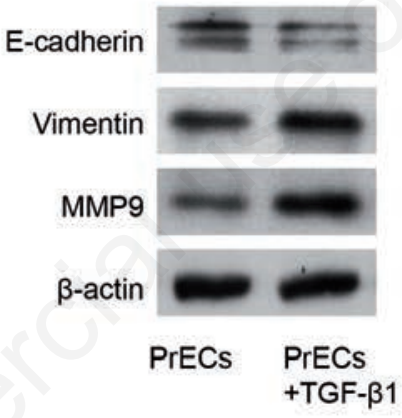

f d

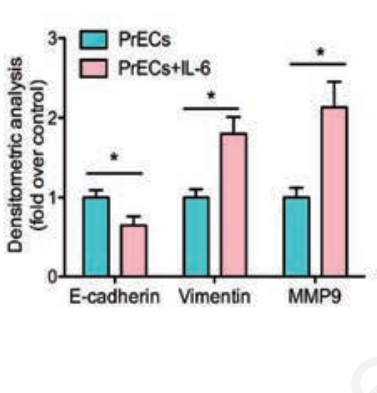

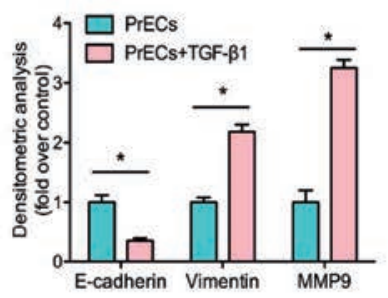
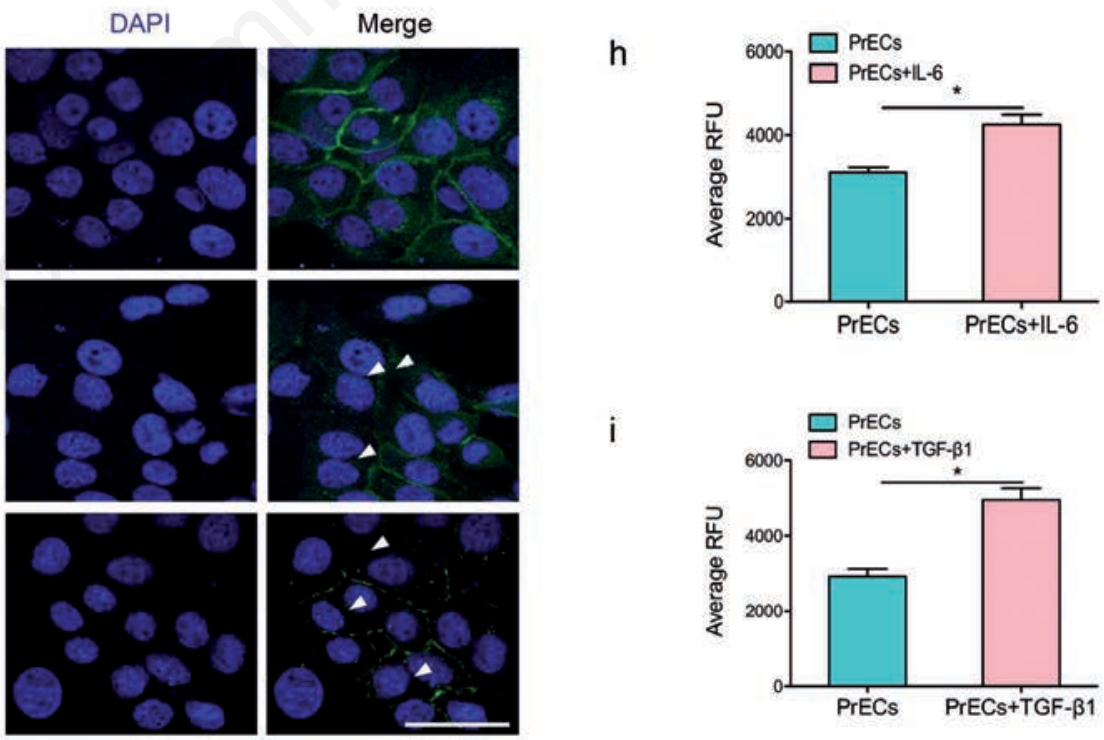

Figure 2. IL-6 and TGF- $\beta 1$ induce EMT features by decreasing E-cadherin expression (a,b) qRT-PCR analysis of Cdh1, vimentin, and MMP9 mRNA levels in PrECs and IL-6 $(50 \mathrm{ng} / \mathrm{mL})$ or TGF- $\beta 1(5 \mathrm{ng} / \mathrm{mL})$-treated PrECs. c) Western blot showed the expression of Ecadherin, Vimentin, and MMP9 protein levels in PrECs and IL-6 or (e) TGF- $\beta 1$-treated PrECs. d,f) The band intensity corresponding to E-cadherin, Vimentin, and MMP9 was quantified and normalized to $\beta$-actin using ImageJ software. $g$ ) Immunostaining of E-cadherin (green, white arrowhead) expression in PrECs and IL-6 or TGF- $\beta 1$-treated PrECs; scale bar: $50 \mu \mathrm{m}$. $\mathrm{h}$ ) PrECs and IL-6 or (i) TGF- $\beta 1$ treated PrECs were allowed to migrate on collagen-coated wells and were stained with calcein AM; the fluorescence intensity was measured. All of the results are representative of at least three independent experiments. The data are expressed as the mean $\pm S D,{ }^{*} \mathrm{P}<0.05$. All of the P values were based on Student's t-test. 


\section{IL-6 and TGF- $\beta 1$ induce DNA}

hypermethylation at the promoter region of $\mathrm{Cdh} 1$

We further investigated the hypothesis that the down-regulation of IL-6 and TGF$\beta 1$-induced E-cadherin levels were associated with the changed DNA methylation level of the Cdh1promoter region, because when
Cdh1 was inhibited by specific siRNA, the protein expression of EMT features, Vimentin and MMP9 were increased in PrECs and BPH1 cells (Figure 3 a-d). Besides, when Cdh1 was inhibited by specific siRNA, cell migrating on collagen coating were significantly promoted (Figure 3e). Then, bisulfite sequencing PCR (BSP) methods were used to examine the DNA methylation level of the Cdh1 promoter region. The DNA sequence of the Cdh1 promoter $(-1000 \mathrm{bp}$ to $0 \mathrm{bp}$ upstream of the transcription start site) was analyzed for $\mathrm{CpG}$ sites and $\mathrm{CpG}$ island (Figure 3f). In total, a DNA sequence including $10 \mathrm{CpG}$ sites within the $\mathrm{CpG}$ island (from -185 bp to $-57 \mathrm{bp}$ ) was amplified (Figure 3e, black box) for BSP analysis. The results showed a

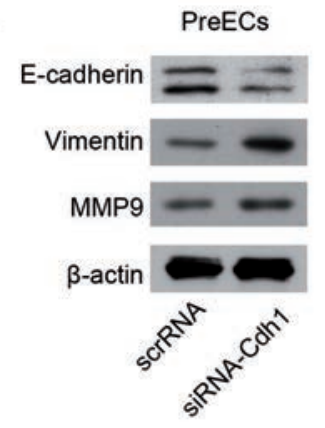

e

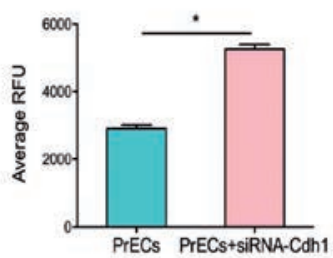

b

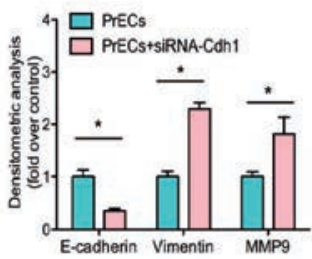

C

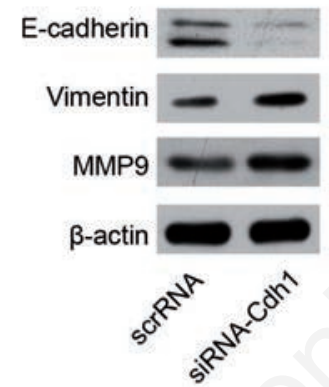

d

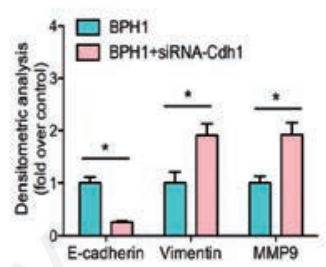

f

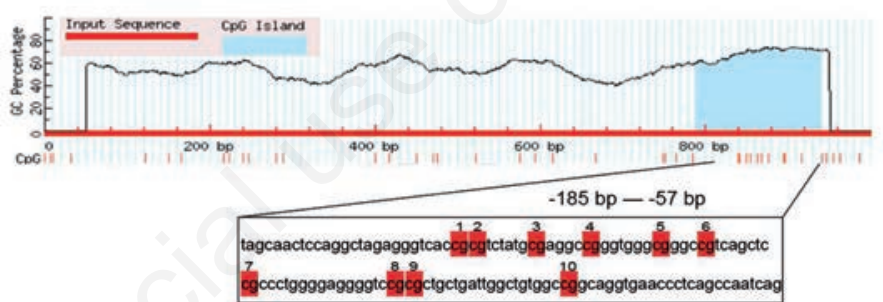

$\mathrm{h}$

\section{㟧}

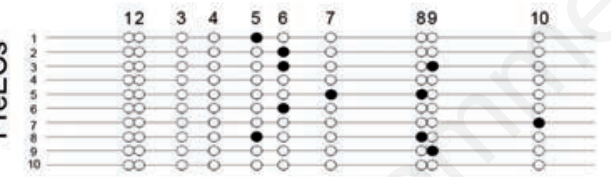

岕
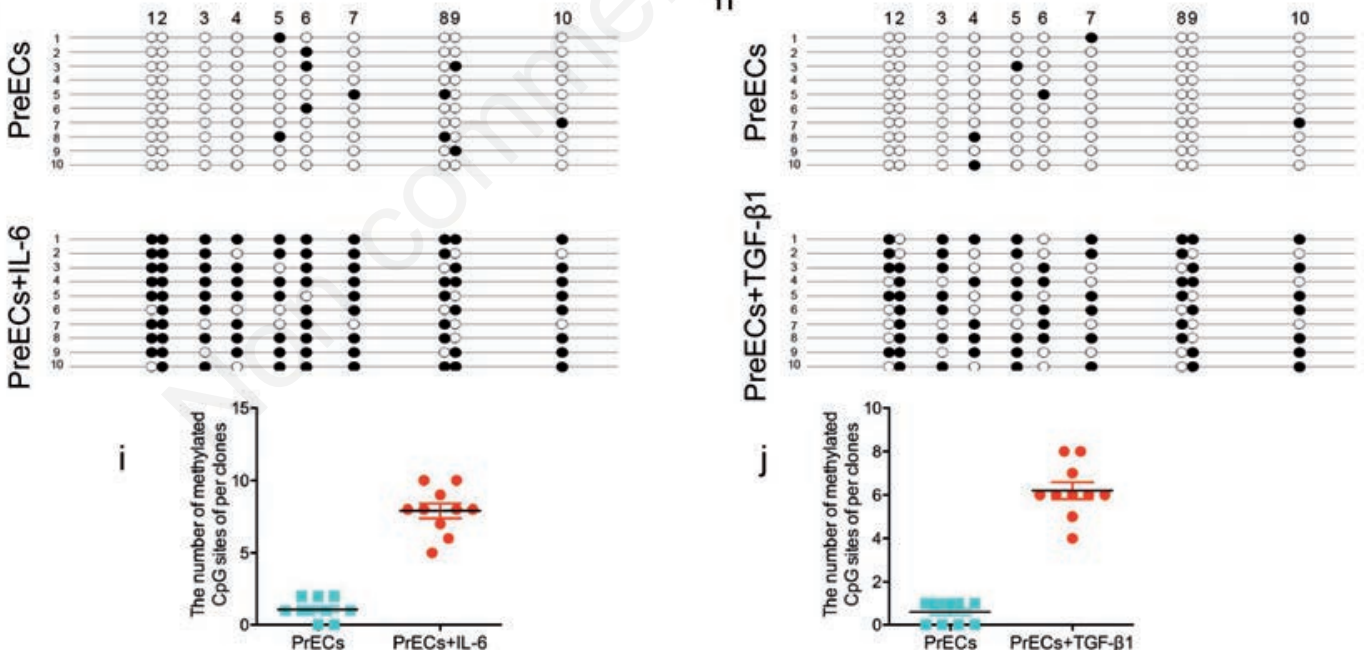

Figure 3. IL-6 and TGF- $\beta 1$ induce DNA hypermethylation at the promoter region of Cdh1. a) Western blot analysis of E-cadherin, Vimentin, and MMP9 protein levels in PrECs and (c) BPH1 cells after transfection with specific Cdh1-siRNA or its corresponding scrambled control (scrRNA). b,d) The band intensity corresponding to E-cadherin, Vimentin, and MMP9 was quantified and normalized to $\beta$-actin using ImageJ software. e) PrECs and siRNA-Cdh1-treated PrECs were allowed to migrate on collagen-coated wells and were stained with calcein AM; the fluorescence intensity was measured. f) The DNA sequence of the Cdh1 promoter region was analyzed for $\mathrm{CpG}$ sites and CpG islands; the black box indicates the DNA sequence $(-185 \mathrm{bp}$ to $-57 \mathrm{bp}$, the transcription start site was indicated as 0 ) of the $\mathrm{CpG}$ island that we selected for further analysis of DNA methylation; a total of $10 \mathrm{CpG}$ sites was included (numbered 110). g,h) Bisulfite sequencing PCR showed that exposure to IL- 6 and TGF- $\beta 1$ significantly increased the methylated CpG sites of the Cdh 1 promoter region; each row represents an individual allele that was randomly cloned and sequenced; circles represent $\mathrm{CpG}$ sites analyzed (10 sites per allele): black circle, methylated $\mathrm{CpG}$ site; white circle, unmethylated $\mathrm{CpG}$ site. $\mathrm{i}, \mathrm{j})$ The number of $5 \mathrm{mC}$ sites was compared between PrECs and IL-6 or TGF- $\beta 1$-treated PrECs. The Mann-Whitney U-test was used to assess the distributional differences of variance across different test samples. ${ }^{*}$ Mann-Whitney $U$-test, $\mathrm{P}<0.01$. The median values are represented by horizontal bars. All results are representative of at least three independent experiments. The data are expressed as the mean $\pm S D, * P<0.05$. 
that exposure to IL- 6 and TGF- $\beta 1$ for $72 \mathrm{~h}$ significantly increased the methylated $\mathrm{CpG}$ sites of the Cdh1 promoter region (Figure 3 $\mathrm{g}, \mathrm{h})$, and the number of methylated $\mathrm{CpG}$ (5 $\mathrm{mC}$ ) sites was quantified (Figure $3 \mathrm{i}, \mathrm{j}$ ).

DNMT1 modulates IL-6 and TGF-

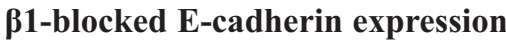

After finding that IL-6 and TGF- $\beta 1$ induced DNA hyper-methylation at the promoter region of Cdh1, we further explored the causal relationships between DNA methylation and the IL- 6 and TGF- $\beta 1$ blocking of E-cadherin expression. Because it was reported that Dnmt1, not Dnmt3a or Dnmt3b, regulated methylation of the SRD5A2 gene promoter in the aging adult prostate, ${ }^{8}$ we focused on the role of Dnmt1.
The expression of Dnmt1 in PrECs and BPH1 cells was measured by RT-PCR and western blot analysis. Consistent with previous reports, ${ }^{6}$ the expression level of Dnmt1 was higher in BPH1 than PrECs (Figure 4 a,b).

Furthermore, the DNMT1 activity measured in the nuclear extracts of BPH1 cells was higher than in PrECs (Figure 4c),

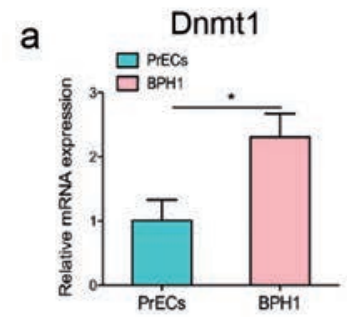

e

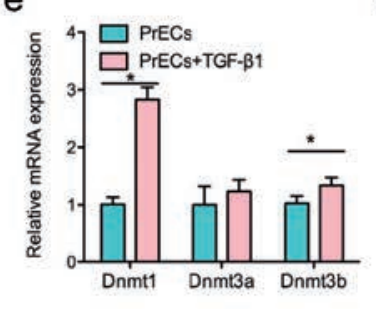

b

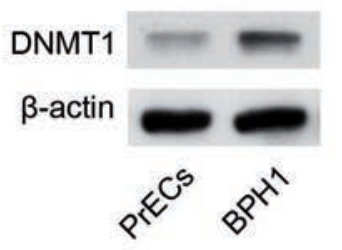

f

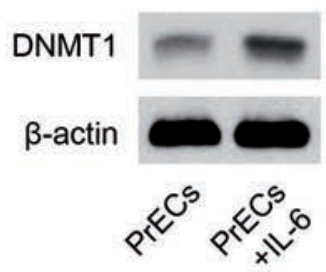

C

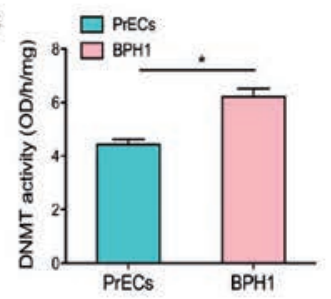

g

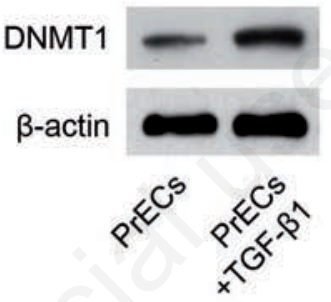

d

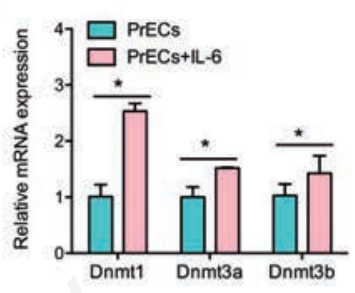

h

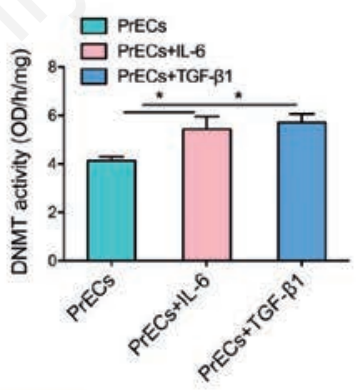

i
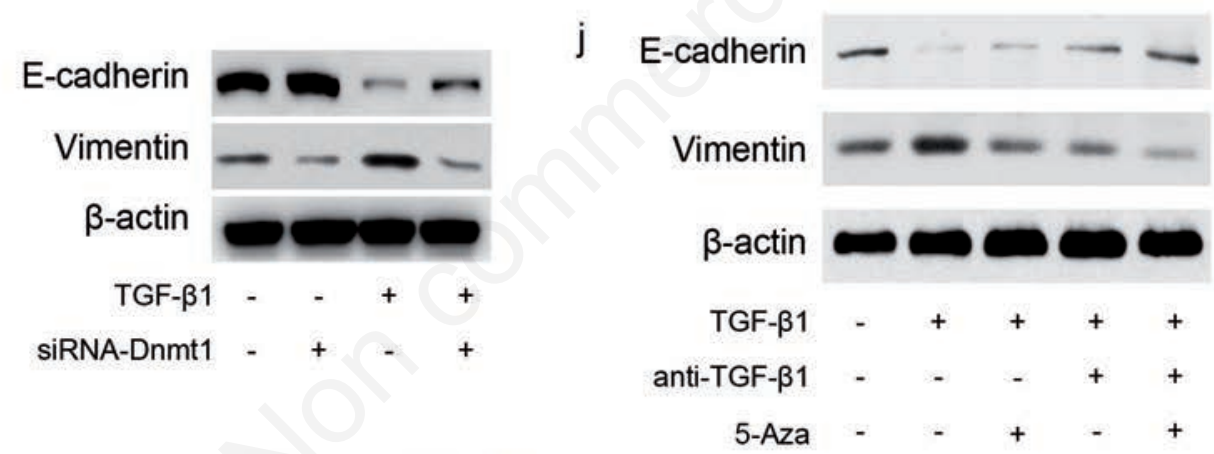

k

PreECs+TGF- $\beta 1+5-A z a$

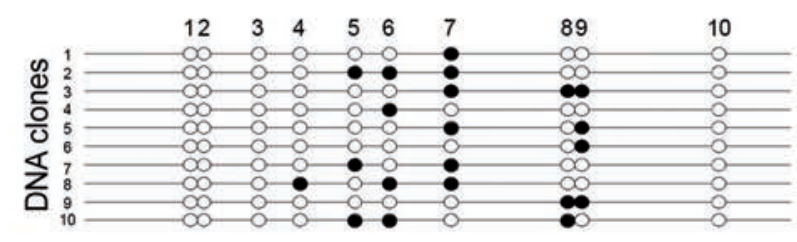

I E-cadherin

Vimentin

MMP9

$\beta$-actin

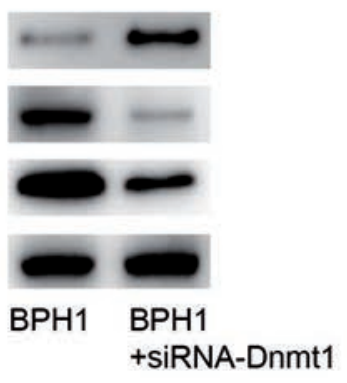

Figure 4. DNMT1 modulates IL-6 and TGF- 31 -blocked E-cadherin expression. a) qRT-PCR and (b) Western blot analysis of DNMT1 between PrECs and BPH1 cells. c) DNMT1 activity measured in the nuclear lysates of PrECs and BPH1 cells. d,e) qRT-PCR analysis of Dnmt1, Dnmt3a, and Dnmt3b between PrECs and IL-6 or TGF- $\beta 1$-treated PrECs. f,g) Western blot analysis of DNMT1 among PrECs and IL-6 or TGF- $\beta 1$-treated PrECs. h) DNMT1 activity measured in the nuclear lysates of PrECs and IL-6 or TGF- $\beta 1-$ treated PrECs. i,j) Western blot analysis of E-cadherin, Vimentin, and MMP9 protein levels in PrECs treated with TGF- $\beta 1$, siRNA-Dnmt1, 5Aza (a DNA methyltransferase inhibitor), and anti-TGF- $\beta 1$ antibody. $k$ ) BSP revealed the DNA methylation of the Cdh1 promoter region in PrECs treated with 5-Aza and TGF- $\beta 1$. 1) Western blot analysis of E-cadherin, Vimentin, and MMP9 protein levels between BPH1 and siRNA-Dnmt1-treated BPH1 cells. All of the results are representative of at least three independent experiments. The data are expressed as the mean $\pm S D, * P<0.05$. All $P$ values were based on Student's $t$-test. 
indicating up-regulated methyltransferase functional activity. The expression of Dnmt3a and Dnmt3b in PrECs exposed to IL- 6 and TGF- $\beta 1$ was also studied. We found that the fold change of Dnmt1 expression was much higher than Dnmt3a and Dnmt3b after exposure of PrECs to IL6 and TGF- $\beta 1$ (Figure 4 d,e). Next, after exposure of PrECs to IL- 6 and TGF- $\beta 1$, the protein expression level and methyltransferase activity of DNMT1 were also increased (Figure $4 \mathrm{f}-\mathrm{h}$ ). Then, inhibitors of Dnmt1 5-aza-2'-deoxycytidine (5-Aza), TGF- $\beta 1$ antibodies and specific siRNA for Dnmt 1 were used. The results showed that the TGF- $\beta 1$-blocked E-cadherin and upregulated Vimentin levels were found to have returned to the control group level following treatment with siRNA for Dnmt1 (Figure 4i), 5-Aza or TGF- $\beta 1$ antibodies (Figure $4 \mathrm{j}$ ). BSP further revealed that the TGF- $\beta 1$-induced DNA hyper-methylation level of the Cdh1 promoter region was obviously inhibited by 5-Aza (Figure 4k). In BPH1 cells, we found that the EMT features were also down-regulated by siRNADnmt1, with increased expression of E-cadherin, decreased Vimentin and MMP9 (Figure 41). These results indicated that it was DNMT1 that modulated IL-6 and TGF$\beta 1$-blocked E-cadherin expression by inducing DNA hyper-methylation.

\section{Discussion}

Our study demonstrates for the first time that Dnmt1 had the effect of regulating the pro-inflammatory factors IL-6 and TGF- $\beta 1$ induced EMT in normal PrECs. Further investigations in our study suggested that epigenetic modification might play substantial roles in the development of BPH via DNA methylation of the promoter region of E-cadherin. The issue of whether chronic inflammation influences the initiation and progress of BPH has been a popular topic. ${ }^{17,18}$ Inflammation is acknowledged as one of the potential contributing factors in the prostates of patients with BPH. ${ }^{19}$ Increased infiltration of immune cells and pro-inflammatory factors have previously been found in $\mathrm{BPH}$ and prostate cancers. ${ }^{8}$ High IL-6 concentrations were associated with increased BPH risk in men aged $<65$ years. ${ }^{20}$ Serum IL- 6 and TGF$\beta 1$ were significantly elevated in patients with metastatic prostatic carcinoma, ${ }^{18,21}$ and TGF$\beta 1$ was shown to regulate the proliferation and differentiation of stromal cells in BPH. ${ }^{22}$ However, anti-inflammatory agents, such as steroids and cyclooxygenase-2 (COX-2), used in the treatment of various prostate diseases have limited efficiency and uncertain mechanisms. ${ }^{6,23}$ In this study, we revealed the effects of the pro-inflammatory factors IL-6 and TGF- $\beta 1$ on normal prostatic epithelial cells. IL-6 and TGF- $\beta 1$-blocked E-cadherin expression could be partly rescued by DNMT1 inhibitor. Therefore, the limited efficiency of anti-inflammatory agents in the treatment of $\mathrm{BPH}$ could result from the varied expression and activity of endogenous DNMT1in individual patients. Recently, DNA methylation has been recognized as a dynamic regulator of the development and disease procession of the prostate. ${ }^{24}$ During the development of the prostate, DNA methylation also contributes to prostate bud outgrowth, at least in part by downregulating Cdh1 expression. ${ }^{24,25}$ As a key regulator of EMT, Cdh1 expression is inappropriately silenced by DNA methylation in breast cancers $^{26}$ and prostate cancer. ${ }^{27}$ Since inhibition of Cdh1 is associated with tumor invasion and is predictive of poor patient outcomes, treatment with a DNA methylation inhibitor, which restored Cdh1 expression, decreased invasion activity in prostate cancer cell lines. ${ }^{27}$ The expression of androgen receptor, a vital factor for the developing prostate and the progression of prostate cancer, was regulated by DNA methylation. ${ }^{12,24}$ Regarding $\mathrm{BPH}$, it was shown that increased DNMT1 expression and hyper-methylation of the promoter regions of tumor suppressor genes were observed in BPH tissues, compared with histologically normal prostate tissues. ${ }^{28}$ In the present study of $\mathrm{BPH}$, our results showed that the expression of Dnmt1 was higher in BPH1 cells. DNA hypermethylation at the promoter region of Cdh1 was observed after IL- 6 and TGF- $\beta 1$ treatment, resulting Cdh1 expression being silenced, consistent with the results of prostate cancer cells.

TGF- $\beta 1$ plays a vital role in the initiation of EMT, and its biological mechanisms have been widely studied. ${ }^{29}$ It has been demonstrated to be an inducer of EMT in various normal and cancer cell types. Using prostatic samples from BPH patients, it was found that three key downstream mediators in TGF- $\beta$ signaling - Smad3, Snail, and Slug - were upregulated in $\mathrm{BPH}$ tissue. ${ }^{11}$ Starsichova et al. found that TGF- $\beta 1(10 \mathrm{ng} / \mathrm{mL})$ treatment could drive EMT in BPH1 cells in vitro. ${ }^{29}$ The biological mechanisms of TGF- $\beta 1$ could also be mediated by canonical Smad-dependent signaling and non-canonical signaling pathways. Chen et al. showed that TGF- $\beta 1$ induced EMT through non-canonical PI3K/AKT and MAPK/ERK1/2 signaling pathways. The canonical Smad pathway of TGF- $\beta 1$ signaling can be enhanced by
LPS/TLR4 signaling through the down-regulation of a member of the TGF- $\beta$ type I receptor family, BAMBI (bone morphogenic protein and activin membrane-bound inhibitor).$^{30}$ However, the biological mechanism of TGF- $\beta 1$ was also found to induce global changes in DNA methylation during the epithelial-to-mesenchymal transition in ovarian cancer cells. ${ }^{31}$ This effect of TGF$\beta 1$ was mediated by increased expression and activity of Dnmts, and Dnmts inhibitor reversed the TGF- $\beta 1$-induced mesenchymal phenotype. In prostate cancers, TGF- $\beta 1$ was identified as activating Erk and therefore Dnmts, which could result in promoter DNA hypermethylation of its own receptors. ${ }^{16}$ Similarly, our results showed that TGF- $\beta 1$ treatment indeed induced EMT features and promoted the migration activity of PrECs. Increased expression and activity of DNMT1 were observed in TGF- $\beta 1$ treated PrECs. Thus, TGF- $\beta 1$ activated Dnmt1 might function as an important modulator, independent of canonical Smad-dependent signaling and non-canonical signaling pathways.

Finally, some limitations of our study should be mentioned. First, our findings in epigenetic modifications were limited to DNA methylation. Other epigenetic modifications, particularly histone modifications, should be explored further. It has been revealed that polycomb repressor group 2 (PRC2), which modulates trimethylation of K27 on histone H3 (H3K27me3), suppresses Cdh $1 .{ }^{32,33}$ We speculated that Smad3 or other transcription factor, actived by TGF- $\beta$ signaling, might recruit and bind with Dnmtl or other epigenetic modifiers to regulate the expression of E-cadherin. Furthermore, we only examined DNA methylation of the Cdh1 promoter region, so other EMT features, such as N-cadherin, Vimentin and MMP9, should also be fully assessed. Second, we focused on two widely studied pro-inflammatory factors: IL- 6 and TGF- $\beta 1$. The role of other factors, such as IL- 8 and TNF- $\alpha$, should also be investigated in inducing EMT because it has been shown that EMT could be induced by TNF- $\alpha$ through the NF- $\kappa B$ signaling pathway. ${ }^{34}$ To more accurately simulate the progression of $\mathrm{BPH}$, in vivo research should be conducted in the future.

Collectively, our study showed that the pro-inflammatory factors IL-6 and TGF- $\beta 1$ activated Dnmt1 and could directly regulate the expression of Cdh1and EMT in normal prostate epithelial cells. Although more elaborate investigations should be performed to elucidate the complex regulation mechanisms of DNA methylation, inhibitors of Dnmt1 could be potential therapeutic candidates for the treatment of $\mathrm{BPH}$. 


\section{References}

1. Bostanci Y, Kazzazi A, Momtahen S, Laze J, Djavan B. Correlation between benign prostatic hyperplasia and inflammation. Current Opin Urol 2013;23:5-10.

2. Norström MM, Radestad E, Sundberg B, Mattsson J, Henningsohn L, Levitsky V, et al. Progression of benign prostatic hyperplasia is associated with proinflammatory mediators and chronic activation of prostate-infiltrating lymphocytes. Oncotarget 2016; 7:23581-93.

3. Roehrborn CG. Pathology of benign prostatic hyperplasia. Int $\mathrm{J}$ Impot Res 2008;20(Suppl 3):S11-8.

4. Ooi SK, Qiu C, Bernstein E, Li K, Jia D, Yang Z, et al. DNMT3L connects unmethylated lysine 4 of histone $\mathrm{H} 3$ to de novo methylation of DNA. Nature 2007;448:714-7.

5. Arand J, Spieler D, Karius T, Branco MR, Meilinger D, Meissner A, et al. In vivo control of $\mathrm{CpG}$ and non-CpG DNA methylation by DNA methyltransferases. PLoS Genet2012;8:e1002750.

6. Niu Y, Ge R, Hu L, Diaz C, Wang Z, Wu $\mathrm{CL}$, et al. Reduced levels of 5-alpha reductase 2 in adult prostate tissue and implications for BPH therapy. Prostate 2011;71:1317-24.

7. Ge R, Wang Z, Bechis SK, Otsetov AG, Hua $\mathrm{S}, \mathrm{Wu} \mathrm{S}$, et al. DNA methyl transferase 1 reduces expression of SRD5A2 in the aging adult prostate. Am J Pathol 2015; 185:870-82.

8. Bechis SK, Otsetov AG, Ge R, Olumi AF. Personalized medicine for the management of benign prostatic hyperplasia. J Urol 2014;192:16-23.

9. Thiery JP, Acloque H, Huang RY, Nieto MA. Epithelial-mesenchymal transitions in development and disease. Cell 2009; 139:871-90.

10. Grant CM, Kyprianou N. Epithelial mesenchymal transition (EMT) in prostate growth and tumor progression. Transl Androl Urol 2013;2:202-11.

11. Alonso-Magdalena $P$, Brossner $C$, Reiner A, Cheng G, Sugiyama N, Warner M, et al. A role for epithelial-mesenchymal transition in the etiology of benign prostatic hyperplasia. Proc Natl Acad Sci U S A 2009;106:2859-63.

12. Lu T, Lin WJ, Izumi K, Wang X, Xu D, Fang LY, et al. Targeting androgen receptor to suppress macrophage-induced EMT and benign prostatic hyperplasia (BPH) development. Mol Endocrinol 2012;26:1707-15.

13. Slabakova E, Pernicova Z, Slavickova E, Starsichova A, Kozubik A, Soucek K. TGF-beta1-induced EMT of non-trans- formed prostate hyperplasia cells is characterized by early induction of SNAI2/Slug. Prostate 2011;71:1332-43.

14. Sullivan NJ, Sasser AK, Axel AE, Vesuna F, Raman V, Ramirez N, et al. Interleukin-6 induces an epithelial-mesenchymal transition phenotype in human breast cancer cells. Oncogene 2009;28:2940-7.

15. Panchanathan R, Liu H, Choubey D. Hypoxia primes human normal prostate epithelial cells and cancer cell lines for the NLRP3 and AIM2 inflammasome activation. Oncotarget 2016; 7:28183-94.

16. Zhang Q, Chen L, Helfand BT, Jang TL, Sharma V, Kozlowski J, et al. TGF-beta regulates DNA methyltransferase expression in prostate cancer, correlates with aggressive capabilities, and predicts disease recurrence. Plos One 2011;6:e25168.

17. Penna G, Fibbi B, Amuchastegui S, Cossetti C, Aquilano F, Laverny G, et al. Human benign prostatic hyperplasia stromal cells as inducers and targets of chronic immuno-mediated inflammation. J Immunol 2009;182:4056-64.

18. Adler HL, McCurdy MA, Kattan MW, Timme TL, Scardino PT, Thompson TC. Elevated levels of circulating interleukin6 and transforming growth factor-betal in patients with metastatic prostatic carcinoma. J Urol 1999;161:182-7.

19. Kim HJ, Park JW, Cho YS, Cho CH, Kim JS, Shin HW, et al. Pathogenic role of HIF-1alpha in prostate hyperplasia in the presence of chronic inflammation. Biochim Biophys Acta 2013;1832:18394.

20. Schenk JM, Kristal AR, Neuhouser ML, Tangen CM, White E, Lin DW, et al. Biomarkers of systemic inflammation and risk of incident, symptomatic benign prostatic hyperplasia: results from the prostate cancer prevention trial. Am J Epidemiol 2010;171:571-82.

21. Michalaki V, Syrigos K, Charles P, Waxman J. Serum levels of IL-6 and TNF-alpha correlate with clinicopathological features and patient survival in patients with prostate cancer. Br J Cancer 2004;90:2312-6.

22. Starsichova A, Lincova E, Pernicova Z, Kozubik A, Soucek K. TGF-beta1 suppresses IL-6-induced STAT3 activation through regulation of Jak2 expression in prostate epithelial cells. Cell Signall 2010;22:1734-44.

23. Liu TT, Grubisha MJ, Frahm KA, Wendell SG, Liu J, Ricke WA, et al. Opposing effects of cyclooxygenase-2 (COX-2) on estrogen receptor beta (ERbeta) response to 5alpha-reductase inhibition in prostate epithelial cells. J Biol Chem 2016; 291:14747-60

24. Keil KP, Vezina CM. DNA methylation as a dynamic regulator of development and disease processes: spotlight on the prostate. Epigenomics 2015;7:413-25.

25. Keil KP, Abler LL, Mehta V, Altmann HM, Laporta J, Plisch EH, et al. DNA methylation of E-cadherin is a priming mechanism for prostate development. Dev Biol 2014;387:142-53.

26. Fukagawa A, Ishii H, Miyazawa K, Saitoh M. deltaEF1 associates with DNMT1 and maintains DNA methylation of the E-cadherin promoter in breast cancer cells. Cancer Med 2015;4:125-35.

27. Graff JR, Herman JG, Lapidus RG, Chopra H, Xu R, Jarrard DF, et al. E-cadherin expression is silenced by DNA hypermethylation in human breast and prostate carcinomas. Cancer Res 1995;55:5195-9.

28. Henrique R, Jeronimo C, Hoque MO, Carvalho AL, Oliveira J, Teixeira MR, et al. Frequent 14-3-3 sigma promoter methylation in benign and malignant prostate lesions. DNA Cell Biol 2005;24:264-9.

29. Starsichova A, Kubala L, Lincova E, Pernicova Z, Kozubik A, Soucek K. Dynamic monitoring of cellular remodeling induced by the transforming growth factor-beta1. Biol Proced Online 2009;11:316-24.

30. He Y, Ou Z, Chen X, Zu X, Liu L, Li Y, et al. LPS/TLR4 Signaling enhances TGF-beta response through downregulating BAMBI during prostatic hyperplasia. Sci Rep 2016;6:27051.

31. Cardenas H, Vieth E, Lee J, Segar M, Liu Y, Nephew KP, et al. TGF-beta induces global changes in DNA methylation during the epithelial-to-mesenchymal transition in ovarian cancer cells. Epigenetics 2014;9:1461-72.

32. Cao Q, Yu J, Dhanasekaran SM, Kim JH, Mani RS, Tomlins SA, et al. Repression of E-cadherin by the polycomb group protein EZH2 in cancer. Oncogene 2008;27:7274-84.

33. Herranz N, Pasini D, Diaz VM, Franci C, Gutierrez A, Dave N, et al. Polycomb complex 2 is required for E-cadherin repression by the Snaill transcription factor. Mol Cell Biol 2008;28:4772-81.

34. Li CW, Xia W, Huo L, Lim SO, Wu Y, Hsu JL, et al. Epithelial-mesenchymal transition induced by TNF-alpha requires NF-kappaB-mediated transcriptional upregulation of Twist1. Cancer Res 2012;72:1290-300. 